\title{
New nomograms based on treatment outcome and prognostic factors for patients with spinal diffuse large B-cell lymphoma: A population-based study
}

\section{Ben Wang}

The Second Affiliated Hospital of Wenzhou Medical

\section{Lijie Chen}

Taizhou Hospital of Wenzhou Medical University

\section{Boda Chen}

The Second Affiliated Hospital of Wenzhou Medical

\section{Chenglong Xie}

The Second Affiliated Hospital of Wenzhou Medical

\section{Zhenxuan Shao}

The Second Affiliated Hospital of Wenzhou Medical

\section{Xiaolei Zhang}

The Second Affiliated Hospital of Wenzhou Medical

\section{Jiaoxiang Chen}

The Second Affiliated Hospital of Wenzhou Medical

\section{Xiangyang Wang ( $\nabla$ xiangyangwang@wmu.edu.cn )}

The Second Affiliated Hospital and Yuying Children"s Hospital of Wenzhou Medical University, Zhejiang Spinal Research Center https://orcid.org/0000-0002-6504-2661

\section{Research article}

Keywords: spinal diffuse large B-cell lymphoma, Surveillance-Epidemiology-and End Results database, prognostic factors, survival, nomogram

Posted Date: January 14th, 2020

DOl: https://doi.org/10.21203/rs.2.20787/v1

License: (c) (1) This work is licensed under a Creative Commons Attribution 4.0 International License. Read Full License 


\section{Abstract}

Background: Spinal diffuse large B-cell lymphoma (DLBCL) was a rare and malignant tumor, while few studies researched the prognostic factors. The prognostic factors which might have impacts on spinal DLBCL was not clear. Although chemotherapy was recognized as an optimal treatment method, but the curative effect of radiotherapy and surgery were controversial. Methods: The records of patients with spinal DLBCL were selected from the SEER database from 1991 to 2016 . The incidence obtained by database was analyzed by Joinpoint Regression Program. The optimal cut-off values of age and year of diagnosis were identified by $\mathrm{X}$-tail program. Univariate and multivariate survival analysis were calculated to identify independent prognostic factors. Prognostic factors were included to predict the survival possibility compared with 5 years of overall (OS) and cancer-specific survival (CSS) via the new nomograms. Results: A total of 917 patients were enrolled. Age, year of diagnosis and chemotherapy were demonstrated as independent prognostic factors for CSS and OS, and primary site was another independent prognostic factor for CSS. However, radiotherapy and surgery might be ineffective in survival. All factors were included to generate the nomograms for CSS and OS. The concordance indices (C-index) for internal validation of OS and CSS prediction were 0.697 (95\% Cl: $0.662-0.732)$ and 0.709 (95\%Cl: 0.692-0.727) respectively. Conclusions: Age and year of diagnosis are closely associated with the prognosis of spinal DLBCL, and chemotherapy is an ideal treatment modality. The new nomogram is a favourable tool to evaluate the survival possibility, and is benefit for the oncologist to make clinical decisions.

\section{Background}

Non-Hodgkin's lymphoma (NHL) has been defined as one that is wholly or mainly confined to anatomic sites other than lymph nodes. [1, 2] Primary NHL of the bone (PNHLS) is a rare tumor, accounting for nearly $5 \%$ of malignant bone tumors.[3, 4] Primary NHL of the spine was only between $6 \%$ and $32 \%$ of PNHLS.[5, 6] Even so, according to World Health Organization (WHO) classification and previous papers, diffuse large B-cell lymphoma (DLBCL) of spine still was the most common of lymphoma, accounting for 59.8\%.[7] To our knowledge, the 5-year and 10-year overall survival (OS) of bone lymphoma were $60.6 \%$ and $44.7 \%$ respectively,[3] but the poor prognosis of patients with DLBCL was reported with a 5-year survival rate of $19.17 \%$ and a 10 -year survival rate of $3.33 \%$.[8] And the main clinical feature of spinal DLBCL was nerve compression. Although these patients has been considered to have poor prognosis,[8] the epidemiological characteristics and therapeutic modalities of spinal DLBCL have not been defined in detail, and few studies focus on the prognostic factors because of the rarity of DLBCL. Considering the low incidence and poor prognosis, the study of DLBCL based on a large population is particularly urgent and meaningful. Fortunately, the Surveillance, Epidemiology, and End Results (SEER) database is such a large database. Based on the large population, SEER as an integrated database provides complete information for the study of rare cancers, otherwise the study will be limited by small number of cases.[9, 10] 
The majority of patients with DLBCL occur in thoracic and lumbar spine, while very few DLBCL occur in cervical spine and sacrum.[10] However, previous studies have shown that primary site were not associated with survival of patients with lymphoma,[7] and the similar result also have been found in gastric and sinonasal DLBCL.[11, 12] Age at diagnosis and sex were significantly associated with survival of patients with lymphoma, while the other literature showed no association.[7, 12] Race was a significantly vital predictor of survival for patients with cancer,[13] but it seems to be not correlated with the prognosis of DLBCL.[11, 12, 14] In addition, Ann Arbor stage also might be related to overall or cancerspecific prognosis for DLBCL. Therefore, the demographic characteristics and tumor features as prognostic factors must be included in prognostic analysis. Furthermore, although radiotherapy and chemotherapy both were often used as a routine treatment for DLBCL, the efficacy of radiotherapy for different site of DLBCL always was not clear. Surgery seemed to play a role on patient prognosis in a study, but it seemed to have no effect on prognosis in sinonasal DLBCL.[12] We should try to clarify the curative outcome of various treatment on DLBCL patients.

Therefore, here we selected 917 patients with DLBCL from SRRE database between 1991-2016. The aim of this study was to display the epidemiologic features, characterize the prognostic factors and further use the nomograms to predict survival of patients initially diagnosed with DLBCL.

\section{Methods}

\section{Data source}

All patients with spinal DLBCL were obtained from the National Cancer Institute's SEER program (https://seer.cancer.gov). The SEER database collected cancer data covering nearly $30 \%$ of population in the United States.[15] We sent the agreement form to the SEER administration, then got permission from the administrator. Then we acquired the right to obtain the complete information of patients with personal account from the SEER*Stat 8.3.6 software. All available data in the database were retrospective, so there was no need to require the Institutional Review Board approval in our study.

\section{Patients selection}

From the SEER database, patients diagnosed between 1991 and 2016 were selected by primary spine (C41.2-C41.4) and DLBCL ([ICD-0-3] 9680). We excluded patients with missing follow-up, then a total of 917 patients with spinal DLBCL were selected. In this study, the event outcomes included overall survival (OS) and cancer-specific survival (CSS) by specific codes. CSS was considered as the death as a result of cancer during follow-up, and OS was considered as a result of any cause during follow-up.

Demographic and tumor data from SEER were as follows: age, year of diagnosis, sex (male and female), race (white and other), primary site (pelvic bones, sacrum, coccyx and vertebral column) and Ann Arbor Stage (I, II, III, IV) were obtained. Subsequently, we differentiate treatment modality according to radiotherapy (yes and no), chemotherapy (yes and no) and surgery (yes and no). 
Age and year of diagnosis both were stratified by the X-tile program (Yale University, New Haven, Connecticut, USA),[16] which was used to identified the optimal cut-off values of variables through the minimum $P$ values from log-rank chi-square statistics. Kaplan-Meier (K-M) curves was generated to describe survival time of population, and log-rank test were used to distinguish the differences between survival curves simultaneously.

Data including demographic, tumor data and treatments were incorporated in the univariate log rank analysis for OS and CSS respectively, then statistically significant factors were incorporated in the multivariate Cox proportional hazards model respectively (15). Hazard ratios (HRs) of variables with 95\% confidence intervals $(\mathrm{Cl})$ were showed for OS and CSS. All statistical analyses were performed using SPSS v22.0 (Chicago, IL) and R 3.6.1. Two-sided P-values $<0.05$ were considered to be significant statistically.

The joinpoint regression analysis program (version 4.4.0.0 [National Cancer Institute]) was used to calculate Annual percentage change (APC) and 95\% Cls. Compared with zero, the statistical significance of APCs was determined by $t$ test. In addition, we selected calendar years (joinpoints) to identify the bestfitting log-linear regression model.[17]

Construction and validation of the nomograms

All factors were included to analyze the association between prognostic factors and OS/CSS respectively by Cox proportional hazards regression.[18] Subsequently, we generated new nomograms by R software (version 3.6.1). Heagerty's concordance index (C-index) was calculated to evaluate the predictive performance of the nomogram.

\section{Results}

Baseline characteristics of study population \& incidence

The number of 917 patients with spinal DLBCL were identified between 1991 to 2016 . The annual ageadjusted incidence of DLBCL were displayed in Fig. 1. As can been seen, the trend of incidence of all DLBCL were increased significantly from 1991 to 2003 (APCs: 2.75), and the trend of incidence of spinal DLBCL also were increased significantly from 1991 to 2005 (APCs: 4.55). However, recently, the trend of incidence of all DLBCL was relatively steady from 2004 to 2016 (APCs: -0.6), and the trend of incidence of spinal DLBCL was slightly decreased from 2006 to 2016 (APCs: -3.62). At the same time, the overall and disease-specific survival curve were showed in Fig. 2.

The mean age of diagnosis was 62.9 years, and the median survival time was 113 months (Table 1). As for continuous variables, the optimal cutoff value for age and year of diagnosis were calculated with $X$ tile. In this study, the optimal cut-off value of age was identified as 65 and 75 Year (Supplement $1 \mathrm{~A}$ ). The optimal cut-off value of year of diagnosis was identified as 2009 (Supplement 1 B). Then, an accurate 
grouping was carried out for OS/CSS (Table 1). In the cohort, white patients, patients with occurred in vertebral column and patients with first primary spinal DLBCL were the majority of patients. 
Table 1

Baseline characteristics of patients with spinal diffuse large B-cell lymphoma (diagnosed 1991-2016)

\begin{tabular}{|c|c|c|}
\hline Characteristics & Number of patients & Survival time, Median (IQR), months \\
\hline Median survival time, months $(95 \% \mathrm{Cl})$ & 917 & $113(96.9-129.1)$ \\
\hline Age, $y$, mean \pm SD & 917 & $62.9 \pm 17.2$ \\
\hline \multicolumn{3}{|l|}{ Sex } \\
\hline Female & 413 & $104.0(80.2-127.8)$ \\
\hline Male & 504 & $122.0(96.8-147.2)$ \\
\hline \multicolumn{3}{|l|}{ Year of diagnosis } \\
\hline $1991-2009$ & 583 & $105.0(87.0-123.0 \mathrm{c})$ \\
\hline $2010-2016$ & 334 & NA \\
\hline \multicolumn{3}{|l|}{ Tumor sites } \\
\hline Pelvic bones, sacrum, coccyx & 319 & $120.0(87.4-152.6)$ \\
\hline Vertebral column & 598 & $112.0(94.4-129.6)$ \\
\hline \multicolumn{3}{|l|}{ Race } \\
\hline White & 802 & $110.0(95.3-124.7)$ \\
\hline Other & 113 & $162.0(100.0-224.0)$ \\
\hline Unknown & 2 & NA \\
\hline \multicolumn{3}{|l|}{ Ann Arbor Stage } \\
\hline I & 472 & $120.0(98.4-141.6)$ \\
\hline II & 95 & $128.0(87.7-168.3)$ \\
\hline III & 15 & $139.0(0-348.7)$ \\
\hline IV & 272 & $90.0(59.2-120.8)$ \\
\hline Unknown & 63 & NA \\
\hline \multicolumn{3}{|l|}{ Chemotherapy } \\
\hline Yes & 734 & $140.0(119.8-160.2)$ \\
\hline No & 183 & $9.0(4.75-13.3)$ \\
\hline
\end{tabular}

Abbreviations: NA, not available 


\begin{tabular}{|lll|}
\hline Characteristics & Number of patients & Survival time, Median (IQR), months \\
\hline Yes & 557 & $113.0(94.9-131.1)$ \\
\hline No & 360 & $124.0(90.5-157.5)$ \\
\hline Surgery & & \\
\hline Yes & 234 & $128.0(87.1-168.9)$ \\
\hline No & 678 & $110.0(93.7-126.3)$ \\
\hline Unknown & 5 & NA \\
\hline Abbreviations: NA, not available & & \\
\hline
\end{tabular}

Univariate and multivariate survival analysis for OS

Univariate survival analysis for OS revealed that age, year of diagnosis and chemotherapy were important prognostic factors (Table 2). Multivariate survival analysis for OS revealed that age, year of diagnosis and chemotherapy were independent prognostic factors (Table 2 \& Fig. 3). As can been seen in Fig. 4B, the K-M survival analysis showed that older patients have significantly worse prognosis than younger patients $(P<0.001)$. As shown in Fig. 4D, patients diagnosed between 2010 and 2016 were predicted better outcome than patients diagnosed between 1991 and $2009(P=0.030)$. Furthermore, the treatment of chemotherapy was an important prognostic factor for OS. K-M analysis demonstrated the significant difference between two subsets for OS $(P<0.001)$ (Fig. 4F). 
Table 2

Univariate and multivariate analysis of variables for OS of patients with spinal diffuse large B-cell lymphoma

\begin{tabular}{|c|c|c|}
\hline Subject Characteristics & Univariate & Multivariate \\
\hline$P$ value & $P$ value & OR $(95 \% \mathrm{Cl})$ \\
\hline Age of diagnosis & $\bigotimes 0.001$ & $\bigotimes 0.001$ \\
\hline$\leq 65$ & & 1 (reference) \\
\hline $65-75$ & & 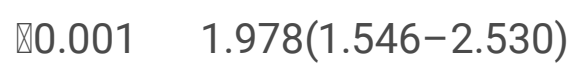 \\
\hline$\otimes 75$ & & 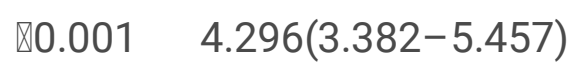 \\
\hline Sex & 0.714 & \\
\hline Race & 0.329 & \\
\hline Year of diagnosis & 0.030 & 0.030 \\
\hline 1991-2009 & & 1 (reference) \\
\hline 2009-2016 & & $0.769(0.607-0.975)$ \\
\hline Tumor sites & 0.258 & \\
\hline Ann Arbor Stage & 0.204 & \\
\hline Chemotherapy & $\varangle 0.001$ & $\llbracket 0.001$ \\
\hline yes & & 1 (reference) \\
\hline no & & $1.978(1.546-2.453)$ \\
\hline Radiation & 0.550 & \\
\hline Surgery & 0.547 & \\
\hline Abbreviations: OS, overa & survival & \\
\hline
\end{tabular}

Univariate and multivariate survival analysis for CSS

According to univariate survival analysis for CSS, these variables, including age, sex, year of diagnosis, primary site and chemotherapy were observed to be associated with prognosis (Table 3 ). Therefore, all of these prognostic factors should be included in multivariate survival analysis for CSS. The result demonstrated that age, year of diagnosis, primary site and chemotherapy were independent prognostic factors for CSS ( $P<0.001, P=0.021, P=0.027$ and $P<0.001$, respectively) (Table 3 \& Fig. 3 \& Fig. 4). However, sex has surprisingly no association with the survival of spinal DLBCL (Table 3 \& Fig. 3). 
Table 3

Univariate and multivariate analysis of variables for CSS of patients with spinal diffuse large B-cell lymphoma

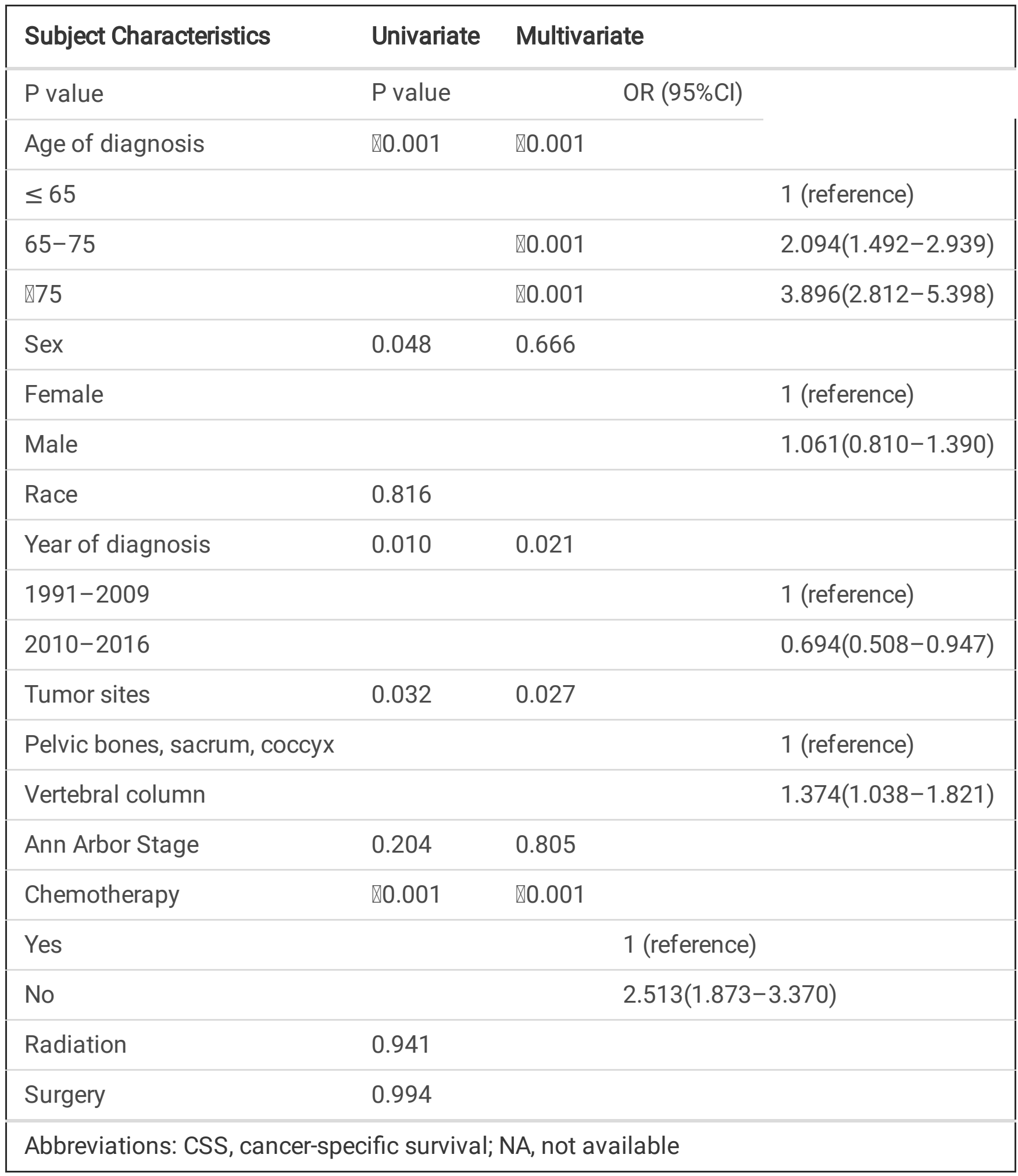

Construction and validation of the nomograms for OS/CSS

Nomograms were constructed for predicting the survival rate of OS/CSS (Fig. 5). According to the nomogram, the value of an individual patient could be located on each variable axis (Red points in 
Fig. 5). Then a line would be drawn upward to match a value of point received for every variable value. The sum of all values was located on the total points axis, and a line would be downward to the survival axes to determine the probability that the patient's survival time was less than 5 years ( 60 months). In addition, the area of blue boxes and yellow area in Fig. 5 showed the number of patients. In the internal validation set, the concordance indices (C-index) for internal validation of OS prediction were 0.697 (95\%Cl: 0.662-0.732), and C-index for internal validation of CSS prediction were 0.709 (95\% Cl: $0.692-$ $0.727)$.

For example, we selected a 57-year-old, married white female with stage IV and first primary tumor of vertebral column, and he received surgery therapy. His total points for CSS was 589 , and the probability of survival time less than 5 year is 0.467 . Besides, his total points for OS was 549 , and the probability of survival time less than 5 year is 0.491 .

\section{Discussion}

DLBCL is the most common subtype of spinal lymphoma, thus it is vital to understand the epidemiology, prognosis and survival prediction of DLBCL. In this study, 917 cases with baseline information were included into the survival analysis. Because of the data based on the large population, we were able to get the incidence trend and calculate the APC from 1991 to 2016. The trend of all and spinal DLBCL both were increased significantly from 1991 to 2003 , while the amplitude of decrease of all DLBCL was not significant in the last ten years. Thus, it is vital and possible to research the prognostic factors and predict the survival possibility of the individual patient by new nomograms.

Despite of the increased incidence, it is delightful that the OS and DSS of spinal DLBCL significantly improved in 2010 to 2016 when compared to 1991 to 2009, suggesting that with current lifestyle and medical conditions, the survival is optimistic in recent years. Except for improving medical care and finance,[19] reducing the incidence of the tumor by intervening independent prognostic factors was also essential. In our study, except for year of diagnosis, we demonstrated age of diagnosis and chemotherapy were significantly associated with the prognosis for OS and CSS, primary site was significantly associated with the prognosis for CSS.

To our knowledge, age was were vital and risk factors for patient with DLBCL in other primary sites.[8, 9, $12,14]$ As expected, younger age had more favorable survival than older age in both univariate and multivariate survival analysis, and the optimal cutoff value was identified as 65 year and 75 year. The KM survival analysis demonstrated that older age was associated with worse prognosis for CSS and OS, with patients more than 75 years having significantly worse prognosis than patients with between 66 and 75 years, with patients under 65 years having better prognosis than patients with between 66 and 75 years.

Han et al reported that sacrum was the rarest primary site.[20] This was represented in this population. In addition, this study showed primary site have significant association with prognosis for CSS, with DLBCL 
occurred in pelvic bones, sacrum and coccyxy having better prognosis. Thus, paying more attention to patients with spinal DLBCL occurred in vertebral column is necessary.

Ann Arbor Stage as commonly staging metric for lymphoma was utilized for DLBCL. In this study, the largest number of patients with recorded staging were those diagnosed stage I, followed by stage IV and II, which was consistent with the previous papers.[12, 21] Race diversity in cancer patients still existed. Black men were diagnosed with more advanced than other races, and might have worse in US.[22] In addition, sex as baseline characteristics often were considered as independent prognostic factors in different bone tumors.[12, 13,23] Except for above baseline characteristics, it is intuitive and possible that the survival time of patients with first primary larger than patients with secondary primary or more. Thus, these factors must be taken into account in the survival analysis. Surprisingly, race, sex, and Ann Arbor Stage were observed no association with the prognosis of OS/CSS in univariate survival analysis. Of course, these factors were not deserved to be included into multivariate survival analysis.

As we all know, DLBCL as one of hematological malignancies, chemotherapy was an optimal treatment. Han et al reported that the majority of patients should receive chemotherapy except individuals whose survival time was too short to receive treatment.[20] Flouzat-Lachaniette et al demonstrated that chemotherapy for DLBCL could significantly shrink the epidural tumor volume.[24] Furthermore, radiation also might be alternative treatment for patients with DLBCL. Peng et al thought the combination of chemotherapy and radiotherapy could efficiently relieve nerve root and spinal cord compression.[25] Several clinicians suggested that the outcome of combination treatment was better than patients treated with radiation or chemotherapy alone. However, the curative effect of radiation lacked statistical support. Considering DLBCL was malignant and aggressive cancer, Han et al observed that surgical decompression significantly improved recovery from neurological deficit.[20] This view was supported by Wang et al, the study showed the 5-year OS rates of patients treated by surgery plus chemotherapy and those received chemotherapy alone were $80.1 \%$ and $49.8 \%$ respectively.[12] However, Binn et al found there was no significant difference between surgery combined chemotherapy and chemotherapy alone. [26], and even Peng et al considered that decompression surgery had negative impact on prognosis.[25] In our study, based on a large population, we first pointed out that chemotherapy, not surgery and radiation, was the independent prognostic factor for spinal DLBCL. In another word, chemotherapy have favorable curative effect on patients, but radiation alone or surgery alone was not associated with the treatment outcome.

By multivariate analysis, new nomograms have been generated to predict individual survival possibility for that it could integrate all prognostic-related factors and could comprehensively evaluate the cumulative effects of factors on tumor patients. In addition, the new nomogram not only could compare the individual's survival time with the 5-year survival (60 months), but also revealed the population propensity in different subgroups and the distribution of prognosis, which was different from traditional nomogram. For example, we gave one case to display. Furthermore, the C-index and $95 \% \mathrm{Cl}$ for CSS and OS showed the ideal validation of survival prediction. 
Of course, several limitations existed in the present study. Firstly, because of limitation of the SEER database, some basic information was not enrolled, including smoking, body mass index, family history. Secondly, we only could obtain the rough treatment. Detail surgical methods and chemotherapy and radiation protocol were not able be acquired. Thirdly, several selection bias might exist in the retrospective trial because of its inherent flaws.

\section{Conclusions}

Spinal DLBCL is a rare aggressive tumor, mainly in male white patients with stage I in vertebral column. Younger age, year of diagnosis and chemotherapy are closely associated with the favorable prognosis for OS. Primary site vertebral column predicts better prognosis for CSS. Surgery and radiation are not an efficient treatment modality. Nomogram is an ideal tool to predict the survival possibility, and is benefit for oncologist to make clinical decisions.

\section{Abbreviations}

DLBCL: diffuse large B-cell lymphoma; NHL: Non-Hodgkin's lymphoma; PNHLS: Primary NHL of the bone; WHO: World Health Organization; OS: overall survival; CSS: cancer-specific survival; SEER: Surveillance, Epidemiology, and End Results; C-index: concordance indices; K-M: Kaplan-Meier; HRs: Hazard ratios; Cl: confidence intervals

\section{Declarations}

\section{Ethics approval and consent to participate}

This study used previously collected deidentified data, which was deemed exempt from review by the Ethics Board of the Second Affiliated Hospital and Yuying Children's Hospital of Wenzhou Medical University.

\section{Consent for publication}

Not applicable.

\section{Availability of data and materials}

The data were abstracted from the Surveillance, Epidemiology, and End Results (SEER) database. This is an open database. (https://seer.cancer.gov).

\section{Competing interests}

The authors declare that they have no competing interests.

\section{Funding}


The present study was sponsored by National Natural Science Foundation of China (81871806).

Authors' contributions

BW, XYW and JXC designed the study. BW and LJC collected the data. BW, BDC and CLX analyzed the data. BW, LJC and ZXS organized the manuscript. XYW and XLZ reviewed the papers and revised the manuscript. All the authors (BW, LJC, BDC, CLX, ZXS, XLZ, JXC and XYW) have read and approved the final manuscript. All authors contributed toward data analysis, drafting and revising the paper and agree to be accountable for all aspects of the work.

Acknowledgements

Not applicable.

\section{References}

1. Baar J, ., Burkes RL, Bell R, ., Blackstein ME, Fernandes B, ., Langer F, . Primary non-Hodgkin's lymphoma of bone. A clinicopathologic study. Cancer 2015, 73(4):1194-1199.

2. Freeman C, Berg JW, Cutler SJ: Occurrence and prognosis of extranodal lymphomas. Cancer 1972, 29(1):252-260.

3. Chisholm KM, Ohgami RS, Tan B, Hasserjian RP, Weinberg OK: Primary lymphoma of bone in the pediatric and young adult population. Hum Pathol 2017, 60:1-10.

4. Glotzbecker MP, Kersun LS, Choi JK, Wills BP, Schaffer AA, Dormans JP: Primary non-Hodgkin's lymphoma of bone in children. $J$ Bone Joint Surg Am 2006, 88(3):583-594.

5. Beal K, Allen L, Yahalom J: Primary bone lymphoma: treatment results and prognostic factors with long-term follow-up of 82 patients. Cancer 2006, 106(12):2652-2656.

6. Ramadan KM, Shenkier T, Sehn LH, Gascoyne RD, Connors JM: A clinicopathological retrospective study of 131 patients with primary bone lymphoma: a population-based study of successively treated cohorts from the British Columbia Cancer Agency. Ann Oncol 2007, 18(1):129-135.

7. Wang Y, Li J, Wei R, Liu C, Nataraj A, Yan J: Prognostic Factors Associated With Bone Lymphoma Primarily Presenting in the Spine. Spine (Phila Pa 1976) 2019, 44(3):185-194.

8. Li S, Wang Z, Wu Z, Zhuang H, Xu Y: Clinical characteristics and outcomes of primary adrenal diffuse large B cell lymphoma in a large contemporary cohort: a SEER-based analysis. Ann Hemato/2019, 98(9):2111-2119.

9. Lin JL, Lin JX, Li P, Xie JW, Wang JB, Lu J, Chen QY, Cao LL, Huang CM, Zheng CH: Dynamic prediction of long-term survival in patients with primary gastric diffuse large B-cell lymphoma: a SEER population-based study. BMC Cancer 2019, 19(1):873.

10. Wang YG, Zhao LY, Liu CQ, Pan SC, Chen XL, Liu K, Zhang WH, Yang K, Chen XZ, Zhang B et al: Clinical characteristics and prognostic factors of primary gastric lymphoma: A retrospective study with 165 cases. Medicine (Baltimore) 2016, 95(31):e4250. 
11. Lin JL, Lin JX, Li P, Xie JW, Wang JB, Lu J, Chen QY, Cao LL, Zheng CH, Huang CM: The Impact of Surgery on Long-Term Survival of Patients with Primary Gastric Diffuse Large B-Cell Lymphoma: A SEER Population-Based Study. Gastroenterol Res Pract 2019, 2019:9683298.

12. Varelas AN, Eggerstedt M, Ganti A, Tajudeen BA: Epidemiologic, prognostic, and treatment factors in sinonasal diffuse large B -cell lymphoma. Laryngoscope 2019, 129(6):1259-1264.

13. Wang B, Chen L, Huang C, Lin J, Pan X, Shao Z, Hu S, Zhang X, Wang X: The homogeneous and heterogeneous risk factors for occurrence and prognosis in lung cancer patients with bone metastasis. J Bone Oncol 2019, 17:100251.

14. Ayub A, Santana-Rodriguez N, Raad W, Bhora FY: Primary appendiceal lymphoma: clinical characteristics and outcomes of 116 patients. J Surg Res 2017, 207:174-180.

15. Duggan MA, Anderson WF, Altekruse S, Penberthy L, Sherman ME: The Surveillance, Epidemiology, and End Results (SEER) Program and Pathology: Toward Strengthening the Critical Relationship. American Journal of Surgical Pathology 2016, 40(12):e94.

16. Camp RL, Marisa DF, Rimm DL: X-tile: a new bio-informatics tool for biomarker assessment and outcome-based cut-point optimization. Clinical Cancer Research 2004, 10(21):7252-7259.

17. Kim HJ, Fay MP, Feuer EJ, Midthune DN: Permutation tests for joinpoint regression with applications to cancer rates. Stat Med 2000, 19(3):335-351.

18. Katz MH, Hauck WW: Proportional hazards (Cox) regression. Journal of General Internal Medicine 1993, 8(12):702-711.

19. Wu J, Sun H, Li J, Guo Y, Zhang K, Lang C, Zou C, Ma H: Increased survival of patients aged 0-29 years with osteosarcoma: A period analysis, 1984-2013. Cancer Med 2018, 7(8):3652-3661.

20. Han S, Yang X, Jiang D, Zhou W, Liu T, Yan W, Wang T, Cheng L, Xiao J: Surgical Outcomes and Prognostic Factors in Patients With Diffuse Large B-cell Lymphoma-associated Metastatic Spinal Cord Compression. Spine (Phila Pa 1976) 2016, 41(15):E943-948.

21. Dubal PM, Dutta R, Vazquez A, Patel TD, Baredes S, Eloy JA: A comparative population-based analysis of sinonasal diffuse large B-cell and extranodal NK/T-cell lymphomas. Laryngoscope 2015, 125(5):1077-1083.

22. J Y, Y Z, X S, AM G, N S, L C, G J, Y H: The prognostic value of multiorgan metastases in patients with non-small cell lung cancer and its variants: a SEER-based study. Journal of cancer research and clinical oncology 2018, 144(9):1835-1842.

23. W Z, Y H, T G, S L, L Y, S W, H C, N W, Y L, W X et al: Application of nomograms to predict overall and cancer-specific survival in patients with chordoma. Journal of bone oncology 2019 , 18(undefined):100247.

24. CH F-L, J A, F R-T, A P: Treatment of spinal epidural compression due to hematological malignancies: a single institution's retrospective experience. European spine journal : official publication of the European Spine Society, the European Spinal Deformity Society, and the European Section of the Cervical Spine Research Society 2013, 22(3):548-555. 
25. Peng X, Wan Y, Chen Y, Chen L, He A, Liao W, Shen J, Fu Q, Han S, Li F et al: Primary non-Hodgkin's lymphoma of the spine with neurologic compression treated by radiotherapy and chemotherapy alone or combined with surgical decompression. Oncol Rep 2009, 21(5):1269-1275.

26. Binn M, Ruskonéfourmestraux A, Lepage E, Haioun C, Delmer A, Aegerter P, Lavergne A, Guettier C, Delchier JC: Surgical resection plus chemotherapy versus chemotherapy alone: comparison of two strategies to treat diffuse large B-cell gastric lymphoma. Annals of Oncology 2003, 14(12):17511757.

\section{Supplemental Figure}

Supplement 1. The optimal cut-off values of age (A) and year of diagnosis (B) were identified by X-tail program.

\section{Figures}

A

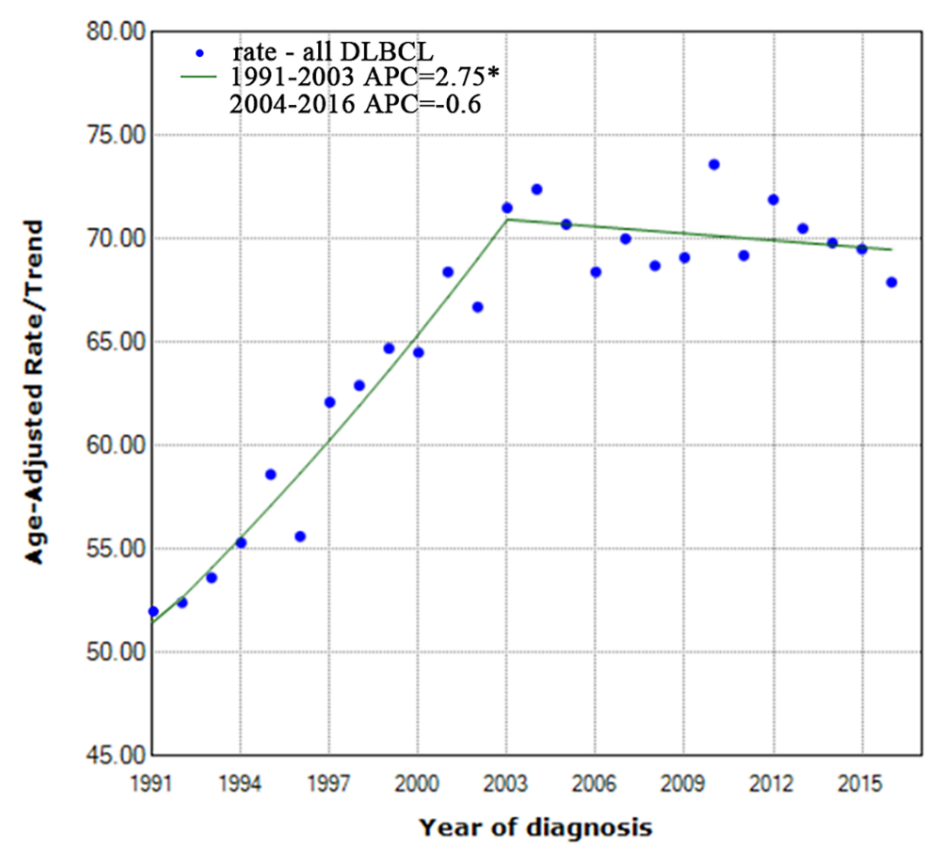

B

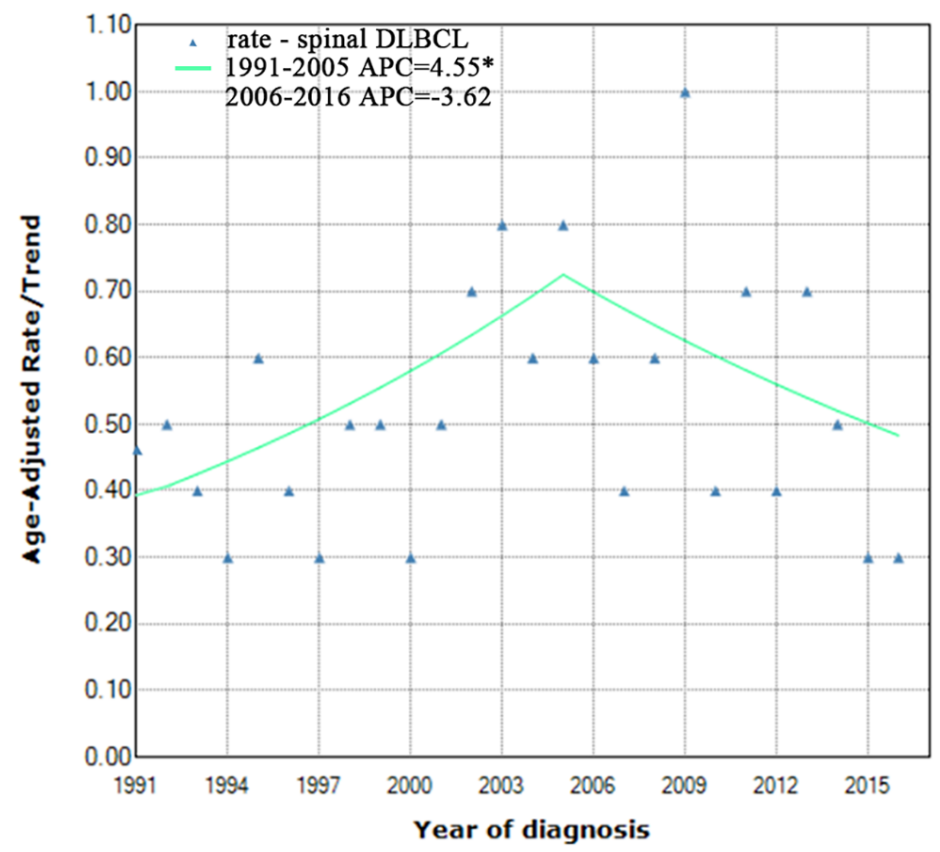

Figure 1

Incidence trend of all (A) and spinal diffuse large B-cell lymphoma (B) between 1991 and 2016. Annual age-adjusted incidence (per 1000000) is illustrated. Abbreviation: APC, annual percentage change; DLBCL, diffuse large B-cell lymphoma 
A

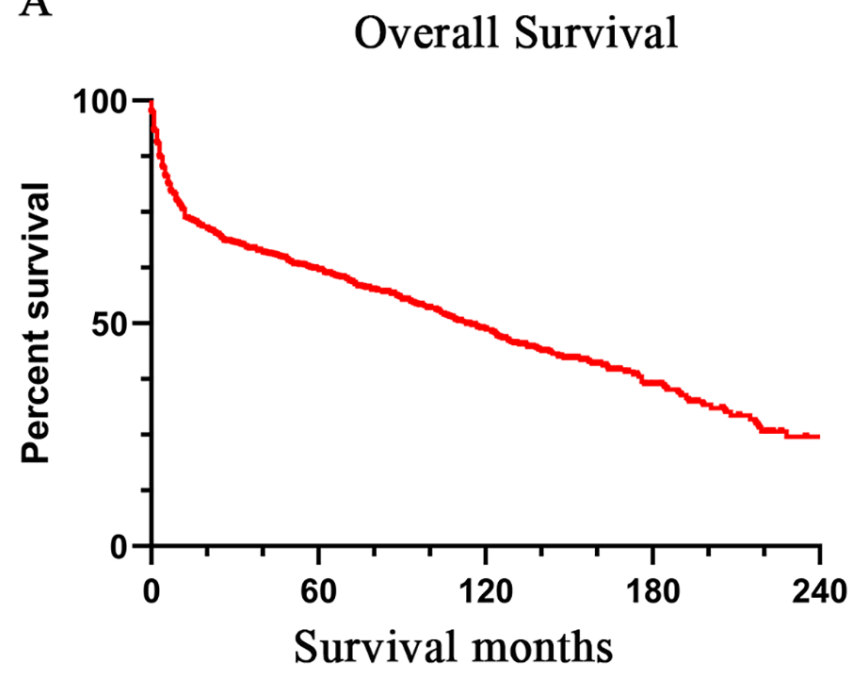

B

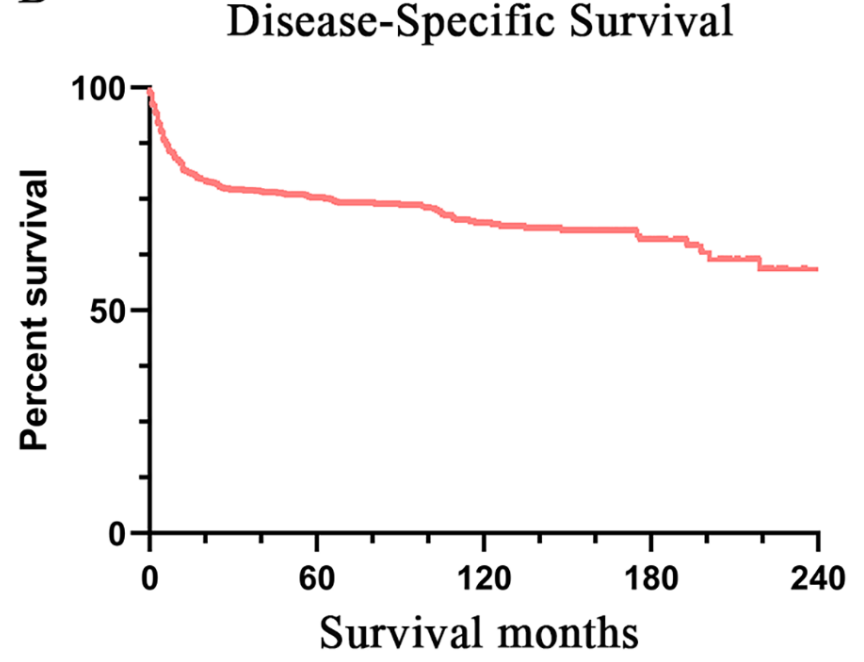

Figure 2

Kaplan-Meier analysis of overall (OS) and cancer-specific survival (CSS). 
Study

ID

ES $(95 \%$ CI $)$

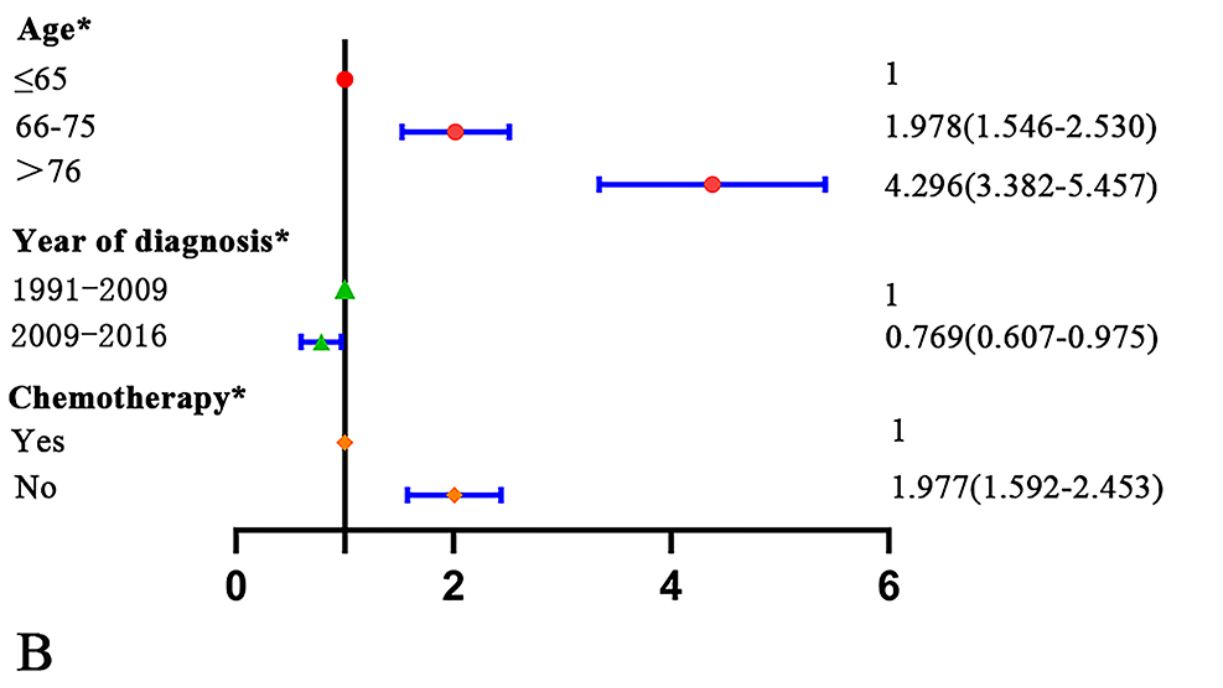

\section{Study}

ID

\section{ES $(95 \% C I)$}

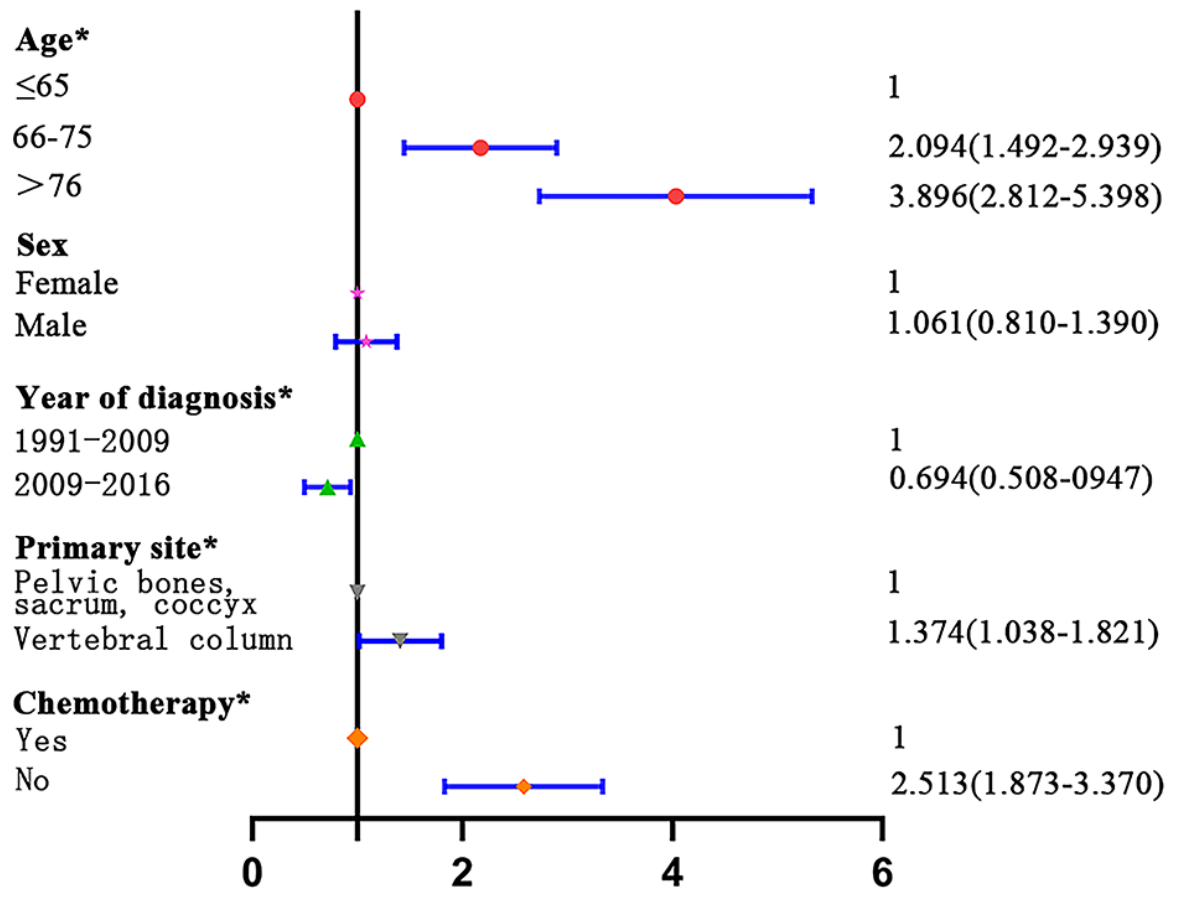

Figure 3

$95 \%$ confidence interval of variables by multivariable regression analysis for overall survival $(A)$ and cancer-specific survival (B) of spinal diffuse large B-cell lymphoma (diagnosed 1991-2016) 
A

CSS for patients stratified by age

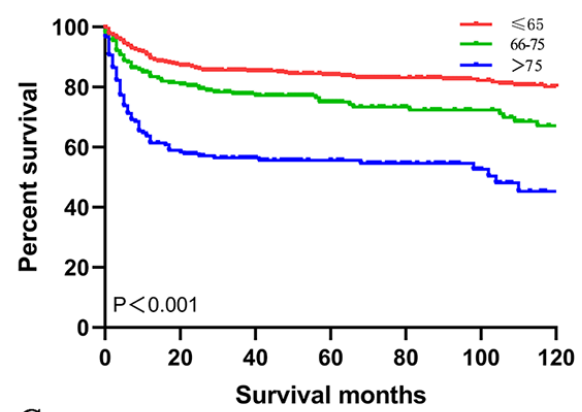

C

CSS for patients stratified by year of diagnosis

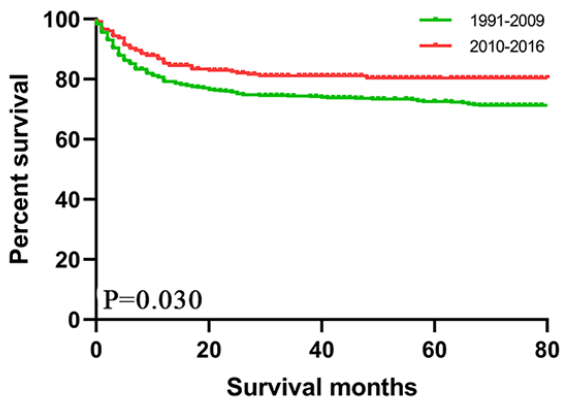

E

CSS for patients stratified by chemotherapy

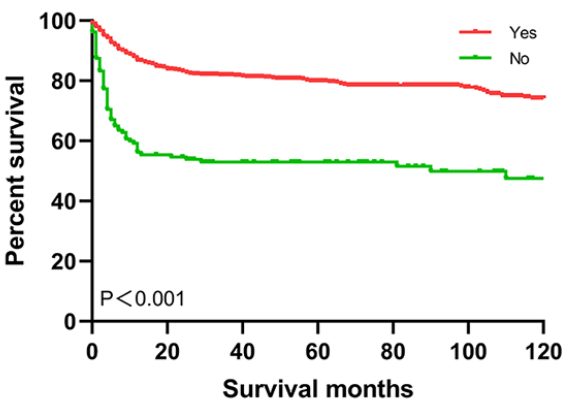

G

CSS for patients stratified by primary site



B

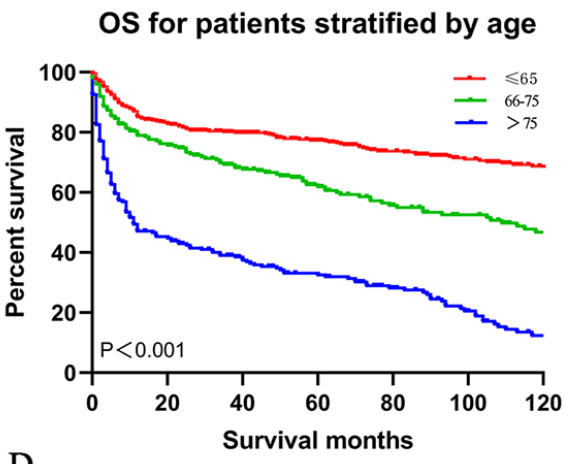

D

OS for patients stratified by year of diagnosis

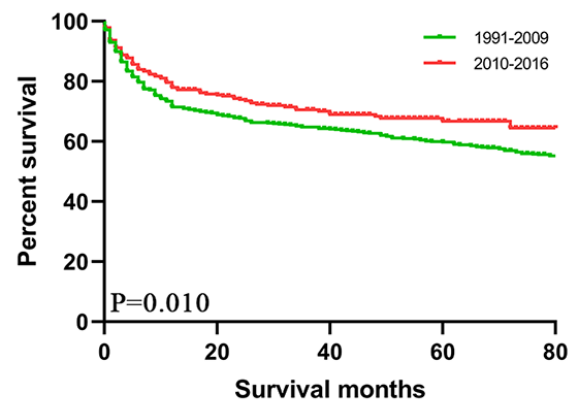

$\mathrm{F}$

OS for patients stratified by chemotherapy

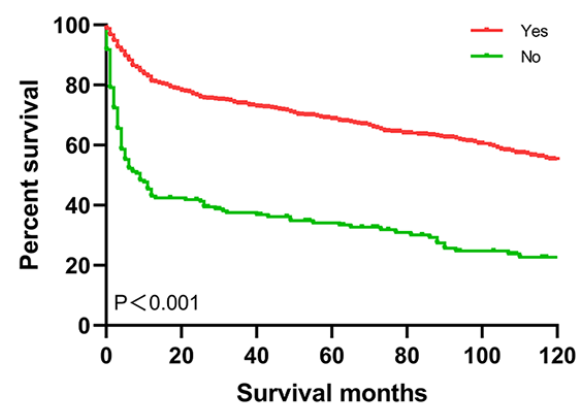

\section{Figure 4}

Survival analysis of cancer-specific survival among patients with spinal diffuse large B-cell lymphoma was stratified by age (A), year of diagnosis (C), chemotherapy (E) and primary site (G). Survival analysis of overall survival among patients with spinal diffuse large B-cell lymphoma were stratified by age (B), year of diagnosis (D) and chemotherapy (F). Abbreviation: CSS, cancer-specific survival; OS, overall survival 
A

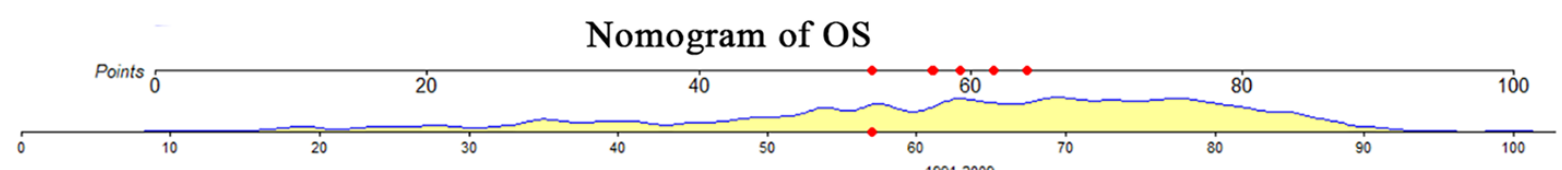

Sex

Race

Site

Stage

Radiation

Chemotherapy ${ }^{\star \star *}$

Surgery

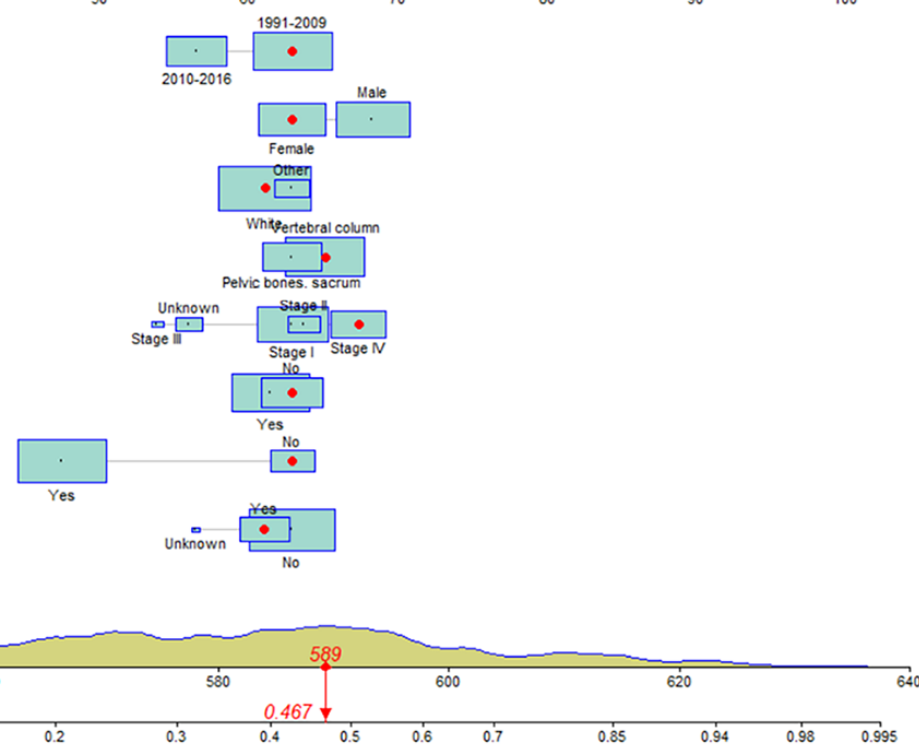

Total-points-to-outcome nomogram:
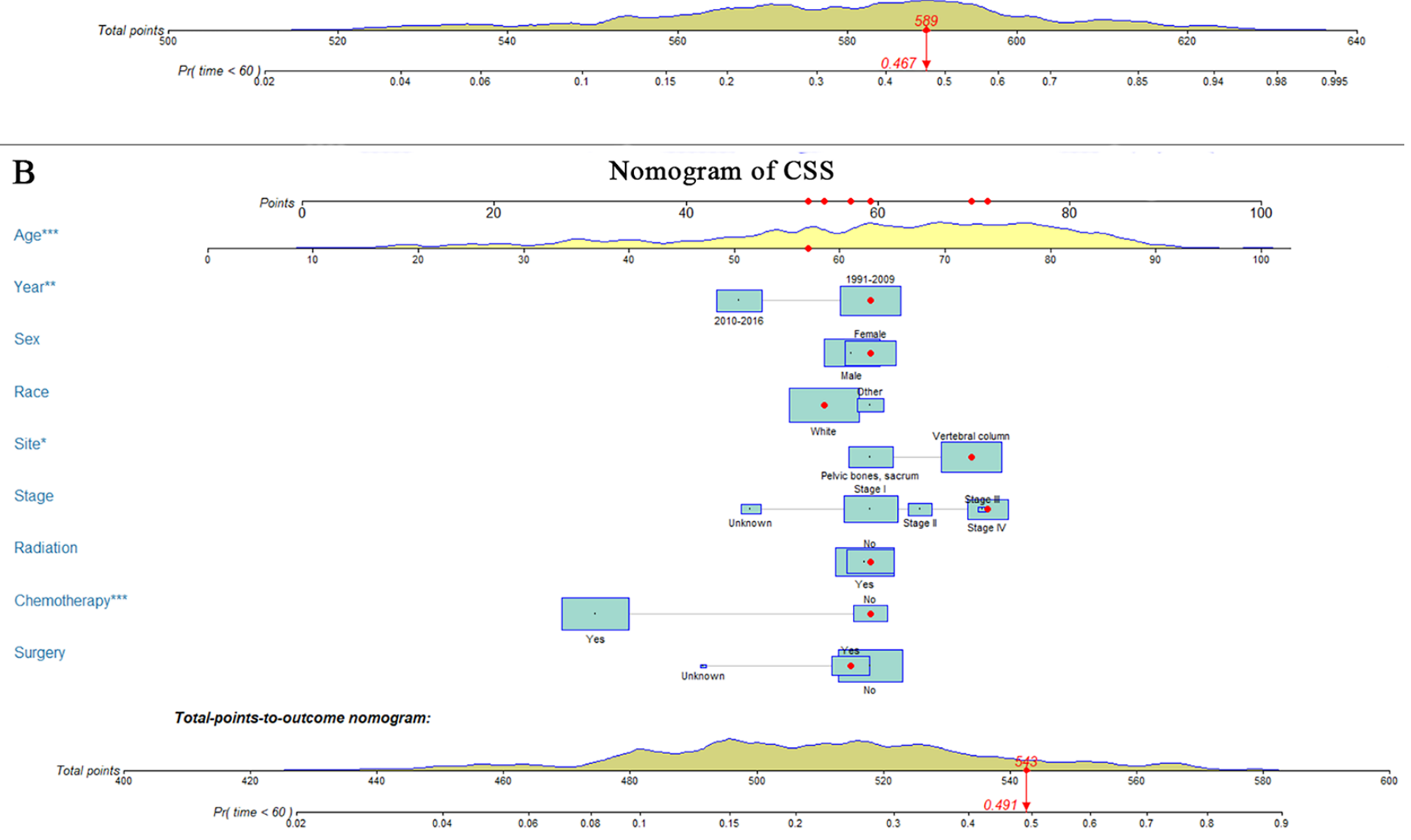

Figure 5

Nomograms were used to predict survival of patients with spinal diffuse large B-cell lymphoma for cancer-specific survival (A) and overall survival (B).

\section{Supplementary Files}


This is a list of supplementary files associated with this preprint. Click to download.

- supplement1.tif 